\title{
Impairment of endochondral ossification by Hoxa2 overexpression: A plausible molecular explanation of idiopathic proportionate short stature
}

\author{
(1:) Pierre M.L. Deprez, ${ }^{1}$ Miloud G. Nichane, ${ }^{2}$ Benoît G. Lengelé, ${ }^{1}$ René Rezsöhazy ${ }^{2}$ and Catherine Nyssen-Behets ${ }^{1}$ \\ UCL 1 Pôle de Morphologie, Institut de Recherche Expérimentale et Clinique, Université catholique de Louvain, Brussels, Belgium \\ 2 Embryologie Moléculaire et Cellulaire Animale, Institut des Sciences de la Vie, Université catholique de Louvain, Louvain-la-Neuve, Belgium
}

Introduction. Using transgenic mice ectopically expressing Hoxa2 all along chondrogenesis, we showed that Hoxa2 exerts a negative influence in the earliest step of endochondral ossification $(1,2)$ and demonstrated that this effect was due to a significant decrease in the number of mesenchymal cells entering chondrogenic differentiation. Furthermore, this endochondral ossification impairment was associated with an overall size reduction phenotype (Fig. 1) which could be referred to human idiopathic proportionate short stature (PSS)

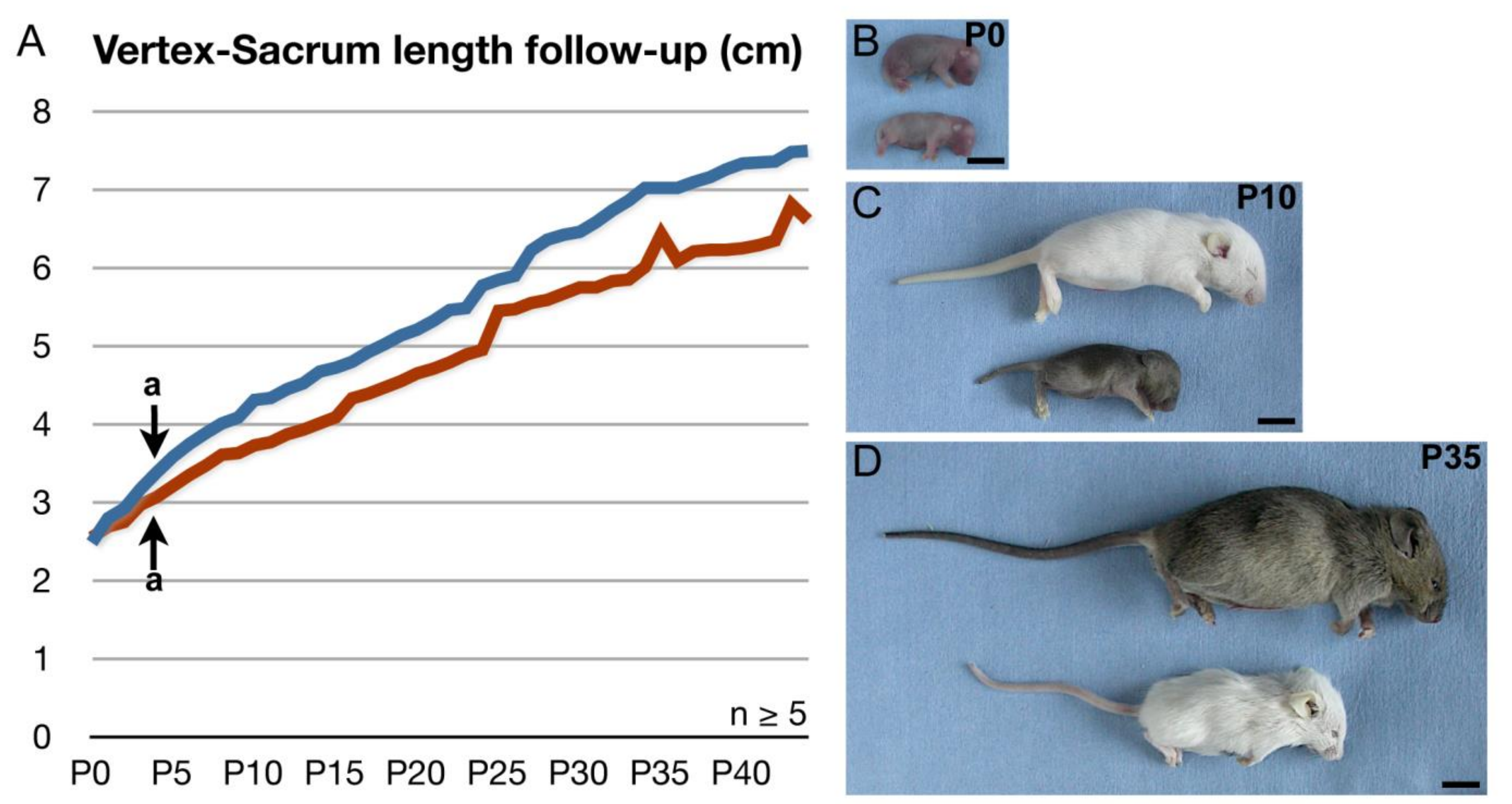

Fig. 1. Hoxa2 expression induces vertex-sacrum length reduction during post-natal life (2). A. Growth curves of BSHoxa2-lacZ (black) and Col2a1-Hoxa2-lacZ (grey). B-D. Pictures of BS-Hoxa2-lacZ (top) and Col2a1-Hoxa2-lacZ (bottom) mice at PO (B) P10 (C) and P35 (D) (Scale bar $=1 \mathrm{~cm}$ ). a $=$ P $<0.05$.

Objective. The molecular pathways underlying the human idiopathic proportionate short stature (PSS) pathogenesis are still unknown. With our murine transgenic model, we here tried to identify the impact of Hoxa2 overexpression on the main factors involved in endochondral ossification in order to evaluate their possible role in idiopathic PSS.

Materials and methods. In our transgenic mice (Col2a1/Hoxa2lacZ; Fig. 2), Hoxa2 expression was induced in Col2a1 expressing territories and maintained thereafter, i.e. all over the endochondral bone elements. Mice bearing the hb-actin-Iox-STOP-Iox-Hoxa2-lacZ transgene only (BS-Hoxa2-lacZ) were considered controls.

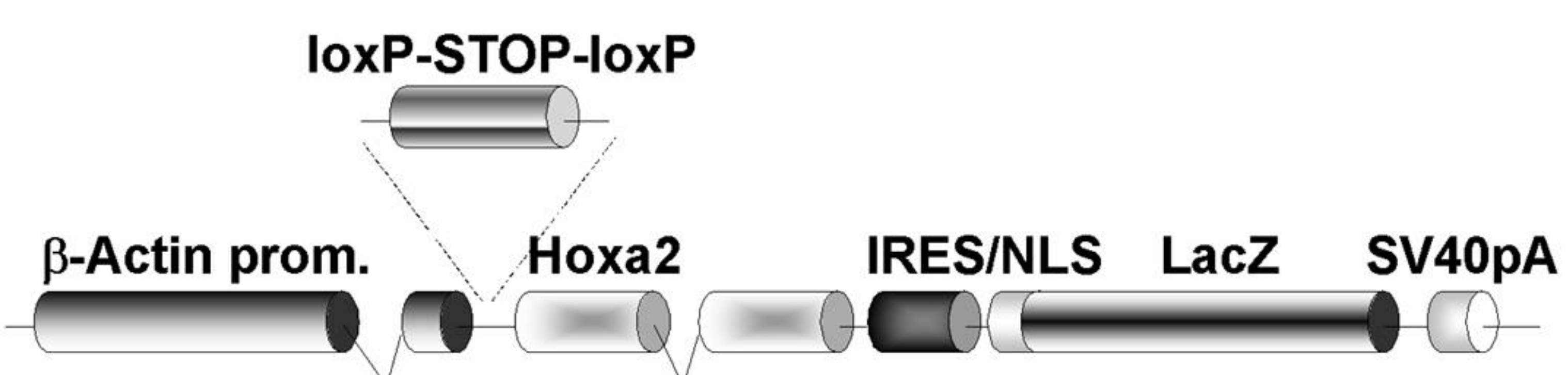

Fig. 2. Construct consists in the Hoxa2 genomic sequence placed under the control of the human $\beta$-actin promoter and translationnally coupled to a lacZ reporter by an Internal Ribosome Entry Site (IRES). It was further modified by inserting a " floxed 》 cassette blocking transcription between the b-actin promoter and the Hoxa2 codin sequence, to give rise to a silent hB-actin-STOP-Hoxa2-IRES-IacZ transgene (BS-Hoxa2-IacZ). Transgenics were mated with mice for a collagen lla1 promoter directed Cre expression (Col2a1-Cre) (3).

Using immunohistochemistry and Western blotting, we compared the protein levels of Bapx1, Runx2, Sox5, Sox6, Bmpr1a, Foxc2, $\beta 1$ integrin, Bmp7, Gdf10, Gdf5, Ihh, Wnt5a, Gdf3, Bmp4, Fgfr3, Gdf6, Meox1, Meox2, Pax1, PthrP, Msx1, Msx2, Osteopontin, Pax9, S-100 and Sox9 in E13.5, E15.5 and E16.5 transgenic and control mice. Immunohistochemistry was performed on sagittal paraffin sections through the spine.

\section{References}

1. Massip L, Ectors F, Deprez P, Maleki M, Behets C, Lengelé B, Delahaut P, Picard J, Rezsöhazy R. Expression of Hoxa2 in cells entering chondrogenesis impairs overall cartilage development. Differentiation 75:256-267, 2007. 2. Deprez PML, Nichane MG, Rousseaux P, Devogelaer JP, Chappard D, Lengelé BG, Rezsohazy R, Nyssen-Behets C. Postnatal growth defect in mice upon persistent Hoxa2 expression in the chondrogenic cell lineage. Differentiation 83: 158-167, 2012

3. Ovchinnikov DA, Deng JM, Ogunrinu G, Behringer RR. Col2a1-directed expression of Cre recombinase in
Results. Persistent expression of Hoxa2 in chondrogenic territories provokes a general down-regulation of several factors known to control the endochondral differentiation cascade, i.e. Sox9, Bapx1, Bmp7, Bmpr1a, Msx1, Pax9, Sox6 and Wnt5a. Furthermore, later molecular agents, such as Ihh and Runx2, are also reduced. As a consequence, Hoxa2 misregulation in mice, which induces a proportionate short stature phenotype mimicking human idiopathic conditions, is associated with a decrease of factors whose effects are distributed along the endochondral ossification cascade.

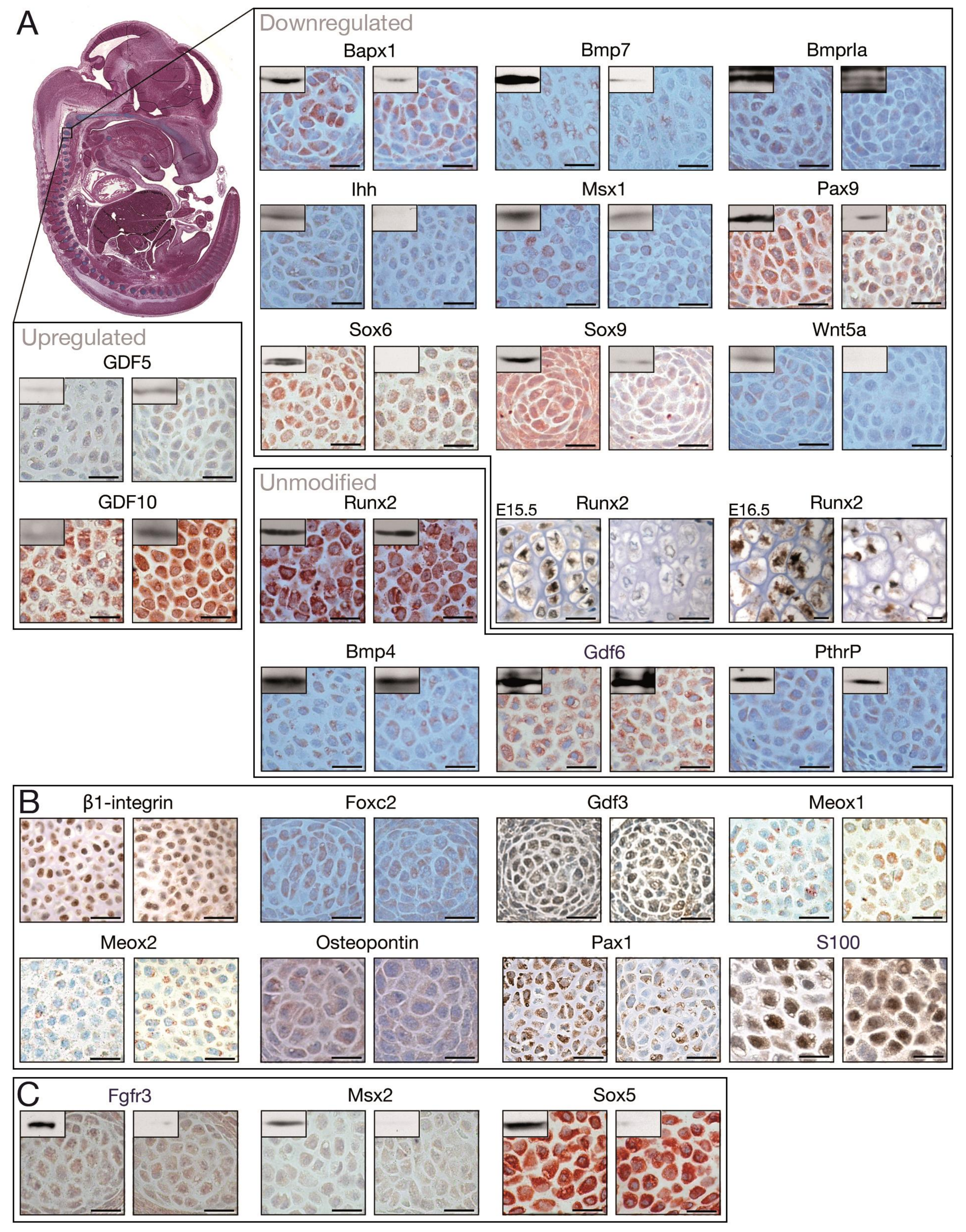

Fig. 3. Immunohistochemistry and western blotting on E13.5 BS-Hoxa2-lacZ (left) and Col2a1/Hoxa2-lacZ (right) vertebral bodies. Consistent results for both techniques are featured in panz $A$. Molects. E13.5 but reduced in pre- and hypertrophic chondrocytes as shown in $E 15.5$ and E16.5 limbs, respectively.

Conclusions. Our results reveal molecular mechanisms linking the activity of Hoxa2 to a negative impact on endochondral skeleton development (Fig. 4). These data give insights for understanding idiopathic proportionate short stature pathogenesis.

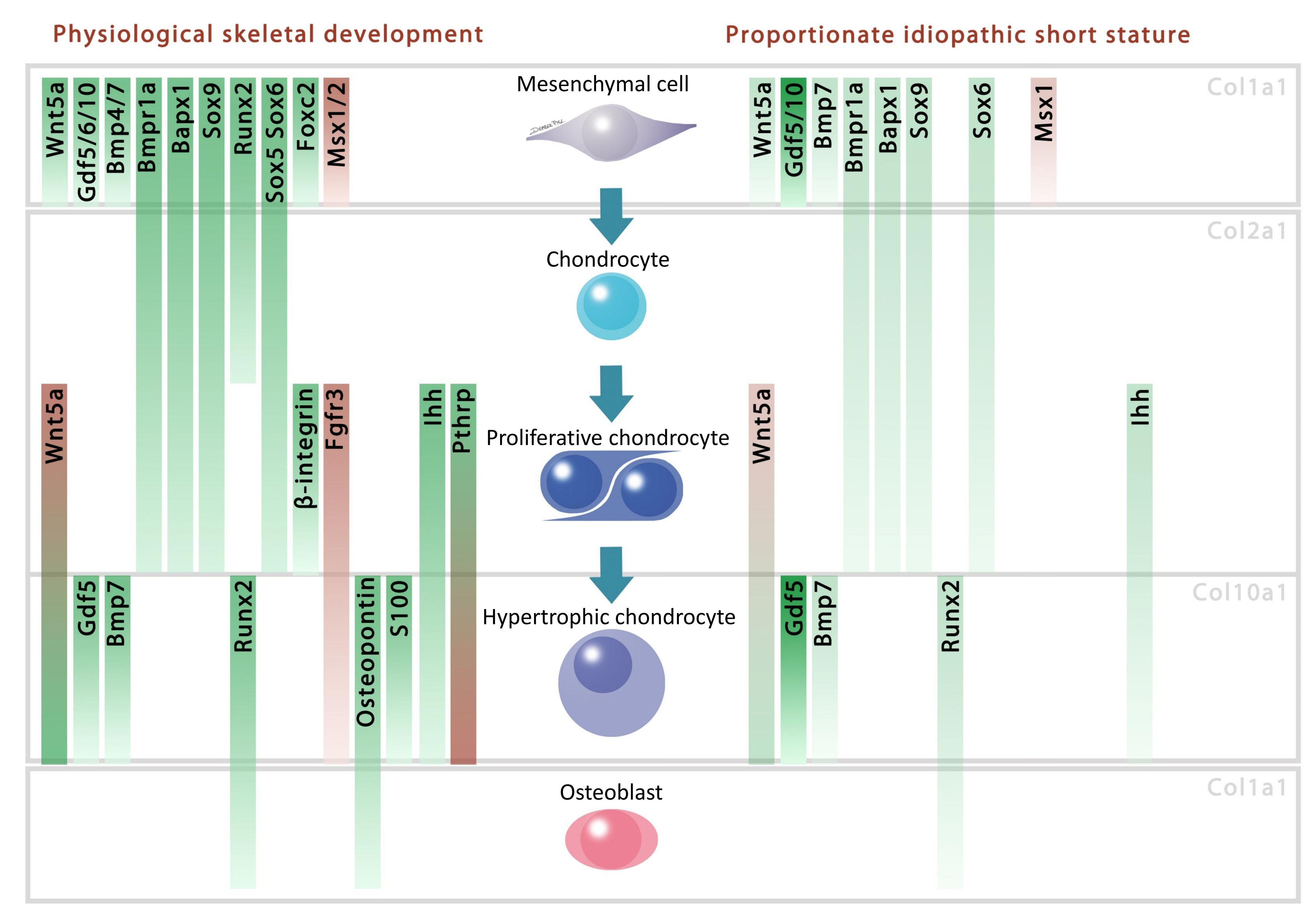

Fig. 4. Model featuring the proposed mechanism leading to idiopathic proportionate short stature. Physiological and pathological molecular expressions are featured on the left and right panels, respectively, according to the mesenchymal (Col1a1), differentiating (Col2a1) and hypertrophic (Col10a1) stages of endochondral ossification. Positive and negative influence of molecules over differentiation and hypertrophy are shown in green and red. Lighter and darker colors highlight inhibition or stimulation respectively, in the right panel. 